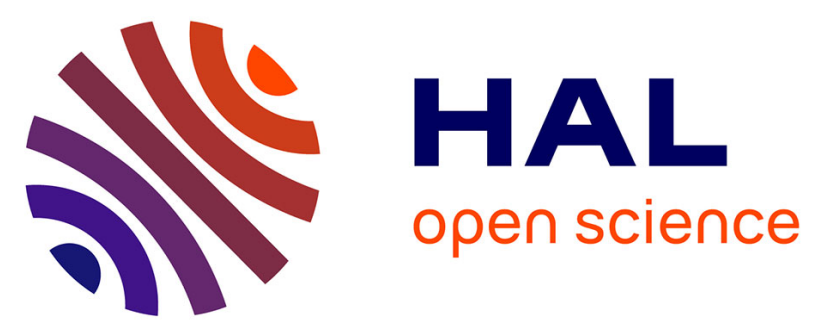

\title{
High-energy chirped- and divided-pulse Sagnac femtosecond fiber amplifier
}

Florent Guichard, Yoann Zaouter, Marc Hanna, Khanh-Linh Mai, Franck Morin, Clemens Hönninger, Eric Mottay, Patrick Georges

\section{To cite this version:}

Florent Guichard, Yoann Zaouter, Marc Hanna, Khanh-Linh Mai, Franck Morin, et al.. High-energy chirped- and divided-pulse Sagnac femtosecond fiber amplifier. Optics Letters, 2015, 40 (1), pp.89. 10.1364/OL.40.000089 . hal-01120352

\section{HAL Id: hal-01120352 \\ https://hal-iogs.archives-ouvertes.fr/hal-01120352}

Submitted on 12 May 2016

HAL is a multi-disciplinary open access archive for the deposit and dissemination of scientific research documents, whether they are published or not. The documents may come from teaching and research institutions in France or abroad, or from public or private research centers.
L'archive ouverte pluridisciplinaire HAL, est destinée au dépôt et à la diffusion de documents scientifiques de niveau recherche, publiés ou non, émanant des établissements d'enseignement et de recherche français ou étrangers, des laboratoires publics ou privés. 


\title{
High-energy chirped- and divided-pulse Sagnac femtosecond fiber amplifier
}

\author{
Florent Guichard, ${ }^{1,2, *}$ Yoann Zaouter, ${ }^{1}$ Marc Hanna, ${ }^{2}$ Khanh-Linh Mai, ${ }^{2}$ Franck Morin, ${ }^{1}$ \\ Clemens Hönninger, ${ }^{1}$ Eric Mottay, ${ }^{1}$ and Patrick Georges ${ }^{2}$ \\ ${ }^{1}$ Amplitude Systemes, 11 avenue de Canteranne, Cité de la Photonique, 33600 Pessac, France \\ ${ }^{2}$ Laboratoire Charles Fabry, UMR 8501, Institut d'Optique, CNRS, Univ Paris Sud 11, 2 Av. A. Fresnel, 91127 Palaiseau, France \\ ${ }^{*}$ Corresponding author: florent.guichard@institutoptique.fr
}

\begin{abstract}
We report on the generation of 1.1-mJ, 300-fs pulses at $50 \mathrm{kHz}$ by implementing an amplifier architecture whereby four stretched pulse replicas are created in the temporal and spatial domains, allowing pulse energy scaling by the same factor. The whole spatiotemporal coherent combining geometry is passive, avoiding the need for active electronic stabilization loop systems. The combining efficiency remains above $90 \%$ at all power levels.
\end{abstract}

OCIS codes: (140.3615) Lasers, ytterbium; (140.3298) Laser beam combining; (320.7090) Ultrafast lasers; (140.3510) Lasers, fiber; (060.2420) Fibers, polarization-maintaining.

High-power femtosecond fiber lasers are now wellestablished as compact and reliable sources of ultrafast pulses. The guiding properties of the amplifying medium guarantees excellent optical-to-optical efficiency and beam quality, but limits the achievable pulse energy because the mode area cannot be scaled arbitrarily. Despite this limitation, femtosecond pulses carrying energy of several microjoules have been generated from such systems [1]. This has been made possible by using very large mode area rod-type fibers and the chirped pulse amplification (CPA) architecture with significant stretching ratios of several nanoseconds. The pulse energy is thereby spread in time and space, limiting the peak intensity that causes detrimental optical nonlinear effects. However, these large stretching ratios are obtained by using very large and expensive diffraction gratings that cannot be reasonably further scaled.

To reach higher energies or reduce the footprint of high energy ultrafast fiber sources, recent experimental efforts have focused on coherent combining of several independently amplified pulses, either in the space or time domains, to further decrease the peak intensity in the amplifying medium. Active coherent beam combining [2], passive coherent beam combining [3], and divided pulse amplification (DPA) $[\underline{4}, \underline{5}]$ have all been recently demonstrated to allow energy scaling. Active parallel beam combination can in principle be scaled arbitrarily, and record performances have been recently obtained using four rod-type fiber amplifiers [6].

In contrast, it is more difficult to scale the number of pulses that can be coherently combined passively, i.e., without the need for active feedback on the optical phase of the pulses. High-energy passive DPA systems [7] are limited by the onset of gain saturation that breaks the symmetry between successive pulse replicas in the amplifiers. This effect limits the number of replicas that can be combined efficiently at high energy to only two [8]. The same is true for passive coherent beam combining [9], because the Sagnac interferometer (SI) only produces two counter-propagating beams. Multiple beams same-path interferometers can in principle be devised, but these architectures are not well suited to high-gain fiber amplifiers due to parasitic laser effect. Scaling the performances of passive coherent combining systems therefore requires the use of spatial and temporal strategies simultaneously. In particular, DPA can be used in conjunction with CPA [8], or with passive coherent combining [9], to allow even further increase of performances.

In this report, we use simultaneously four strategies in the space and time domains to decrease the peak intensity inside $\mathrm{Yb}$-doped amplifying fibers: very large mode area rod-type fibers, CPA, DPA, and passive coherent beam combining embodied in an SI. The full use of passive combining techniques allows the generation of high temporal quality 300 -fs pulses with 1.1-mJ energy at a repetition rate of $50 \mathrm{kHz}$, corresponding to an average power of $55 \mathrm{~W}$. This is achieved by an optical setup with a limited footprint of $120 \mathrm{~cm} \times 60 \mathrm{~cm}$.

The experimental setup is shown in Fig. 1 . The front end is composed of a passively mode-locked ultrafast oscillator, a pulse picker, a stretcher, and a low-power fiber preamplifier. The oscillator generates pulses at the central wavelength of $1030 \mathrm{~nm}$ and pulse duration of $200 \mathrm{fs}$. The incident pulses are stretched to $500 \mathrm{ps}$, and stretching parameters are designed to introduce a dispersion mismatch between the stretcher and the compressor

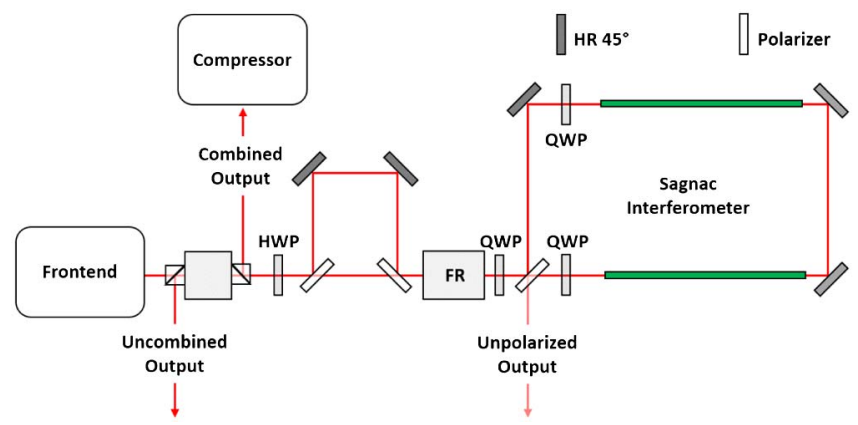

Fig. 1. Experimental setup of the passively combined DCPA Sagnac amplifier. FR, Faraday rotator; HWP, half-waveplate; QWP, quarter waveplate. 
units in order to partially compensate for the residual phase accumulated in the moderately nonlinear main amplifier [10]. The repetition rate of the oscillator is reduced by an acousto-optic modulator pulse picker to obtain a train of pulses at $50 \mathrm{kHz}$. This train of pulses is sent through an isolator that is also used to extract the final amplified and recombined beam. A freespace unbalanced interferometer with polarizers used as splitting and recombining components creates two linearly and orthogonally polarized replicas in the time domain. Depending on the orientation of the input linear polarization, the power fraction sent in each replica can be adjusted. The path length difference of this Mach-Zehnder interferometer corresponds to a delay of $1.2 \mathrm{~ns}$. The divided-pulse beam then propagates through a combination of Faraday rotator and quarter wave plate prior to the SI to ensure proper spatiotemporal recombination on the way back through the system as detailed in [9].

Each time-domain replica is split in two spatially separated beams in the SI. This loop interferometer includes two state-of-the-art ytterbium-doped double-clad rodtype fiber amplifiers. The mode field diameter of each rod is $60 \mu \mathrm{m}$, and their length $80 \mathrm{~cm}$. Furthermore, they are arranged in a completely symmetric geometry to ensure that the spatial properties of the counter-propagating beams to be combined are as identical as possible in order to yield high combining efficiency. Each fiber amplifiers is pumped from the SI splitter/recombiner side with a 180-W diode lasers emitting system at $976 \mathrm{~nm}$ and coupled into $200-\mu \mathrm{m}$ multimode fibers. No additional optical elements were added to prevent reinjection of a pump diode into the other, since the pump light is almost completely absorbed in $1.6 \mathrm{~m}$ of rod-type fibers. Seed coupling from one fiber amplifier to the other is done through relay imaging. The SI also includes two quarterwave plates between the splitter and both fiber inputs to operate the amplifiers in circular polarization, thereby decreasing accumulated optical nonlinearities. After propagating through the SI, the polarization state of each beam is flipped by $90^{\circ}$ and is routed back through the DPA Mach-Zehnder. The overall optical arrangement and polarization evolution results in an identical optical path for all four spatiotemporal replicas, and the pulses coherently combine without the need for active stabilization devices. By design, this architecture is immune to phase noise at frequencies lower than the inverse propagation time in the system, around $10 \mathrm{MHz}$. After extraction by the input polarizer associated to the isolator, the amplified pulses are finally sent to an $85 \%$ efficiency gratings-based compressor.

Possible phase mismatch in space and time between replicas can alter the coherent combining process. This corresponds to a fraction of power being directed toward the uncombined output of Fig. 1. In time, parasitic replicas can appear at delay values corresponding to the DPA Mach-Zehnder path length difference. Experimentally, the combining efficiency is defined as follow:

$$
\eta=\frac{P_{\text {combined }}}{P_{\text {combined }}+P_{\text {uncombined }}},
$$

where $P_{\text {combined }}$ and $P_{\text {uncombined }}$, respectively, correspond to the average power measured along the combined and uncombined output. Moreover in our setup, the SI arrangement leads us to define another residual average power output, denoted as the unpolarized output in Fig. 1. This power fraction is related to polarization evolution effects that occur during propagation in the SI and is not taken into account in the coherent combining efficiency since it is independent on the relative phase mismatch between counter-propagating beams. In particular, residual birefringence in the fiber amplifier leads to cross-phase modulation, imparting a time-dependent polarization state that causes this unpolarized output power to reach $1 \mathrm{~W}$ at the highest pump power.

The combined, uncombined, and compressed powers are plotted as a function of the total pump power in Fig. 2 , along with the combining efficiency. The laser is operated at $50 \mathrm{kHz}$ and the output average power linearly increases, with a slope efficiency of $44 \%$, to finally reach a maximum value of $64 \mathrm{~W}$ before compression, corresponding to an energy of $1.27 \mathrm{~mJ}$. This slope efficiency is calculated with respect to the combined average output power, which constitutes the useful power fraction. Finally, the $85 \%$ efficiency compressor unit leads to the generation of ultrashort pulses with $1.1 \mathrm{~mJ}$ and $55 \mathrm{~W}$ of average power. Without any coherent combining strategy, and using the same stretching ratio and fiber amplifier, the achievable output compressed pulse energy is $350 \mu \mathrm{J}$, limited by optical damage. This illustrates the benefit of passive coherent combining that provides a performance scaling factor in energy very close to the number of replicas.

The maximum combining efficiency of $\sim 94 \%$ is obtained for a combined and compressed average power of $10 \mathrm{~W}$, corresponding to a low accumulated $B$-integral per replica of only $2.4 \mathrm{rad}$. The efficiency then slightly decreases to $90.5 \%$ when the output energy is increased. To maintain the highest possible combining efficiency, a careful adjustment of the half-waveplates that controls the amount of energy sent into each time-domain replica is necessary. This optimization requirement can be understood as follows: as the amplified energy increases, saturation of the gain occurs, leading to temporally

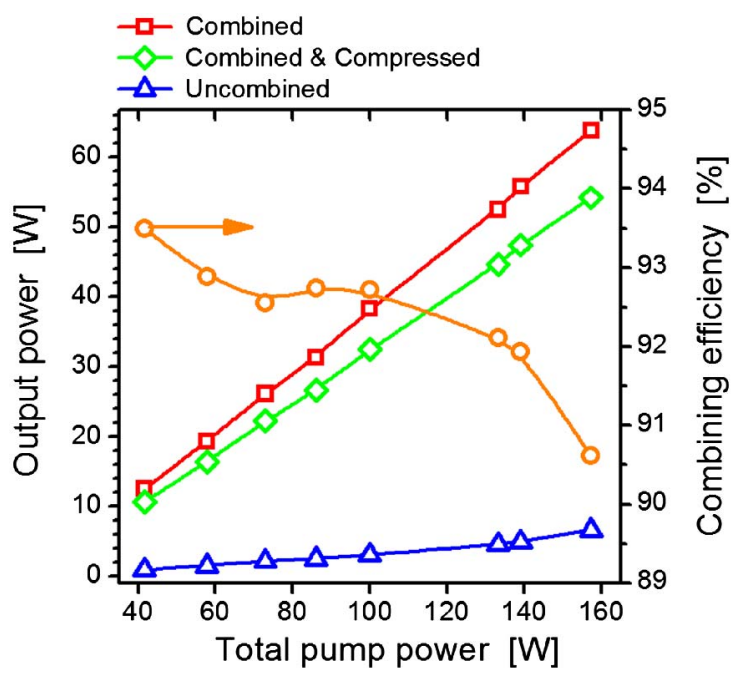

Fig. 2. Combining efficiency measured at $50 \mathrm{kHz}$. Uncombined (blue), combined (red), and compressed (green) average power of DCPA-Sagnac amplifier. 
decreasing gain value. This decreasing energy distribution among pulse replicas in the time domain can in turn be translated into a differential phase by two mechanisms: self-phase modulation and Kramers-Krönig coupling between gain and refractive index along the fiber. This differential phase then hinders proper coherent combining. By adjusting the waveplate, the differential phase can be controlled and leads to optimal DPA combining via the minimization of phase difference between the different temporal replicas to be recombined. It is to be noted that this phase mismatch optimization does not lead to pulse replicas with equal intensities. This effect increases with saturation-dependent differential phase, which explains the drop of combining efficiency at high energies.

In our setup, a phase mismatch between counterpropagating beams can also is translated into a nonrectilinear output polarization state. This can lead to parasitic temporal replicas after propagation in the Mach-Zehnder interferometer, adding a temporal contribution to the combining efficiency. This contribution is defined by the amplitude ratio between the main pulse and the sum of all temporal contributions. The temporal efficiency is measured by sending a portion of the compressed output beam into a fast photodiode and oscilloscope, with an overall bandwidth of $4 \mathrm{GHz}$. Results are shown in Fig. 3 where parasitic replicas are hardly found around the main pulse (see orange curve) with an estimated contribution of less than $1 \%$ at all energy levels when the amplifier is properly optimized. In order to emphasize the temporal position of these replicas, we have unbalanced the SI on purpose, thus generating an important phase mismatch between both beams finally leading to the appearance of clear replicas (blue curve) at $\pm 1.2 \mathrm{~ns}$ around the main pulse. Note that this misalignment would be equivalent to a combining efficiency of less than $60 \%$

Temporal characterizations of the output pulse at maximum energy are carried out with a secondharmonic-generation frequency-resolved optical gating (SHG-FROG) device together with independent

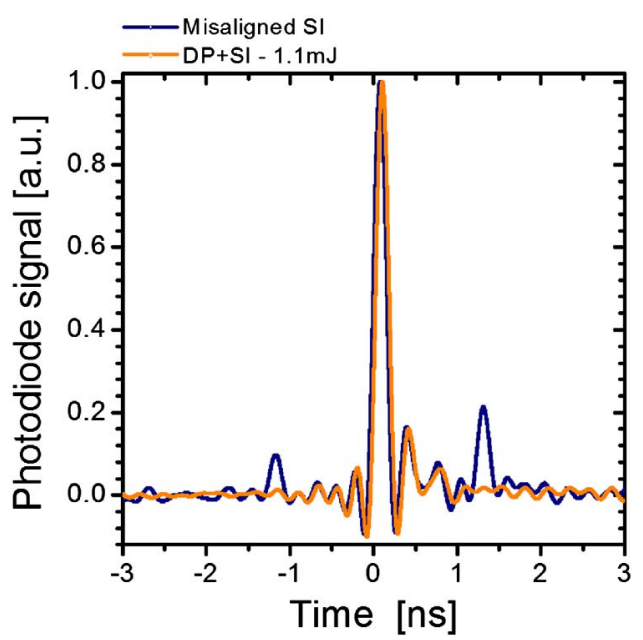

Fig. 3. Combined and compressed temporal traces recorded with a $4-\mathrm{GHz}$ oscilloscope. Blue trace corresponds to an unbalanced SI configuration in order to emphasize the temporal position of parasitic replicas. spectrum and autocorrelation measurements. This data set is plotted in Fig. 4 . Electric field reconstruction is carried out on a $512 \times 512$ grid [see Fig. $4(\mathrm{a})$ ] and quickly converges to a very low FROG error of $22 \times 10^{-4}$. Independent temporal and spectral measurements are in full agreement with the retrieved data and are plotted in Figs. 4(b) and 4(c). The full width at half-maximum (FWHM) of the spectrum is $7.3 \mathrm{~nm}$. The spectral phase is also plotted in Fig. 4(b) (green curve) and shows a residual structure, mainly dominated by a cubic term characteristic of the nonlinear phase compensation induced by the dispersion mismatch between the stretcher and the compressor. In the time domain, the measured and retrieved autocorrelations FWHM are $420 \mathrm{fs}$ [see Fig. 4(c)] corresponding to a retrieved temporal pulse profile FWHM of $300 \mathrm{fs}$. The high-quality retrieved temporal profile is shown in Fig. 3(d) and has a FWHM timebandwidth product of 0.63 . It also exhibits a low-pedestal due to the partial compensation of the nonlinear phase accumulated during amplification. With combined and compressed pulse energy of $1.1 \mathrm{~mJ}$, the retrieved pulse profile has a peak power of $3.1 \mathrm{GW}$.

Finally, we systematically analyze the beam quality at the output of the compressor. High beam quality is expected from the rod-type fibers and the SI as the spatial coherent combining process also acts as a spatial filter for the coherently combined output. The far-field beam profile has a nearly Gaussian shape (see Fig. 5), and $M^{2}$ measurements reveal near diffraction limited values of, respectively, $M_{x}^{2}=1.24$ and $M_{x}^{2}=1.25$ at all energy levels. These values are similar to what is obtained using the same fiber in a standard CPA amplifier.

In summary, we demonstrate that the integration of several passive coherent combining schemes can lead (a)
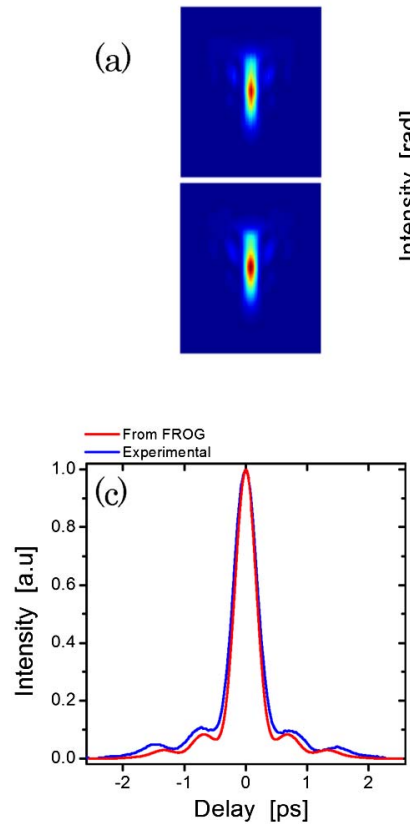
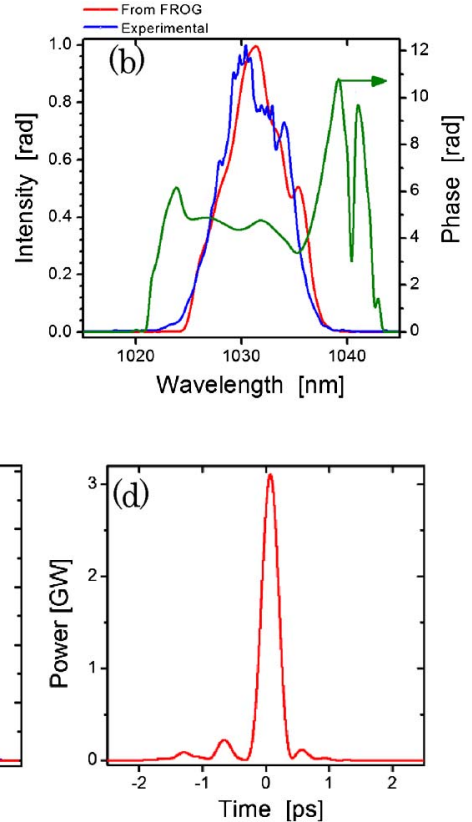

Fig. 4. (a) Measured (top) and retrieved (bottom) FROG trace at $1.1 \mathrm{~mJ}$. (b) Independently measured and retrieved spectrum, green curve corresponds to the retrieved spectral phase. (c) Independently measured and retrieved autocorrelation profiles. (d) Temporal profile and peak intensity of the retrieved pulse. 


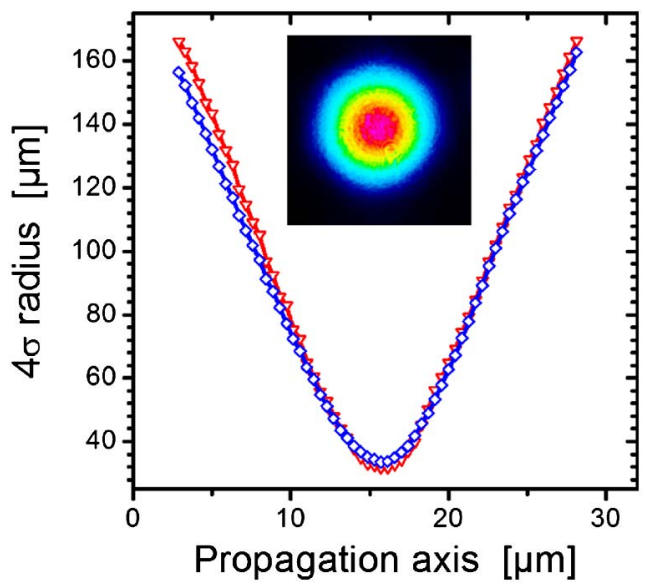

Fig. 5. Combined and compressed 1.1-mJ beam caustic along two transverse orthogonal axis. Insert: far field beam profile.

to the generation of ultrashort multi-GW peak-power pulses from very large mode area rod-type fibers in very compact setups. By implementing the DPA principle before an SI, we generate up to four stretched spatiotemporal replicas of the incoming pulses and scale the output energy by the same factor to generate 300-fs, $1.1-\mathrm{mJ}$ pulses. This is achieved in a moderately nonlinear CPA architecture with stretched pulse durations of only $\sim 0.5$ ns. Therefore, peak power scaling toward $>10 \mathrm{GW}$ can be envisioned by applying multi-ns stretching ratio as in recently published work $[1,11]$. Our fully passive system compares favorably to the active DPA setup that also implements four replicas reported in [12], with similar compressed pulse energy and a higher average power and combining efficiency, although the stretching ratio is 4 times lower in our setup. This illustrates the robustness of the passive combining approach. However, the active approach provides potential scalability in terms of number of replicas that can be used. The average power is currently limited by thermally induced beam pointing drifts that prevent proper combination of the output beams over an extended period of time. This is a technical issue that can be solved with careful thermomechanical design of the optical mounts. Average power scaling is therefore possible because of the separate fiber outputs, enabling power levels beyond the modal instability threshold of a single amplifier.

The authors believe that this laser source is for example an ideal pump source for high repetition rate white-light seeded OPA/NOPA working from UV to mid-IR [13] that are of considerable interest for ultrafast science communities.

\section{References}

1. T. Eidam, J. Rothhardt, F. Stutzki, F. Jansen, S. Hädrich, H. Carstens, C. Jauregui, J. Limpert, and A. Tünnermann, Opt. Express 19, 255 (2011).

2. L. Daniault, M. Hanna, L. Lombard, Y. Zaouter, E. Mottay, D. Goular, P. Bourdon, F. Druon, and P. Georges, Opt. Lett. 36, 621 (2011).

3. Y. Zaouter, L. Daniault, M. Hanna, D. N. Papadopoulos, F. Morin, C. Hönninger, F. Druon, E. Mottay, and P. Georges, Opt. Lett. 37, 1460 (2012).

4. S. Zhou, F. W. Wise, and D. G. Ouzounov, Opt. Lett. 32, 871 (2007).

5. L. J. Kong, L. M. Zhao, S. Lefrancois, D. G. Ouzounov, C. X. Yang, and F. W. Wise, Opt. Lett. 37, 253 (2012).

6. A. Klenke, S. Breitkopf, M. Kienel, T. Gottschall, T. Eidam, S. Hädrich, J. Rothhardt, J. Limpert, and A. Tünnermann, Opt. Lett. 38, 2283 (2013).

7. Y. Zaouter, F. Guichard, L. Daniault, M. Hanna, F. Morin, C. Hönninger, E. Mottay, F. Druon, and P. Georges, Opt. Lett. 38, 106 (2013).

8. M. Kienel, A. Klenke, T. Eidam, M. Baumgartl, C. Jauregui, J. Limpert, and A. Tünnermann, Opt. Express 21, 29031 (2013).

9. L. Daniault, M. Hanna, D. N. Papadopoulos, Y. Zaouter, E. Mottay, F. Druon, and P. Georges, Opt. Express 20, 21627 (2012).

10. Y. Zaouter, J. Boullet, E. Mottay, and E. Cormier, Opt. Lett. 33, 1527 (2008).

11. P. Wan, L.-M. Yeng, and J. Liu, Opt. Express 21, 29854 (2013).

12. M. Kienel, A. Klenke, T. Eidam, S. Hädrich, J. Limpert, and A. Tünnermann, Opt. Lett. 39, 1049 (2014).

13. J. Rothhardt, S. Demmler, S. Hädrich, J. Limpert, and A. Tünnermann, Opt. Express 20, 10870 (2012). 